\title{
Unique treatment potential of cannabidiol for the prevention of relapse to drug use
}

\author{
Friedbert Weiss (iD) and Gustavo Gonzalez-Cuevas ${ }^{1,2}$ \\ Neuropsychopharmacology (2019) 44:229; https://doi.org/10.1038/s41386-018-0218-2
}

Cannabidiol (CBD), the main non-psychoactive and non-addictive constituent of Cannabis sativa, has long received interest as a therapeutic for numerous psychiatric and neurologic disorders (e.g., ref. [1]). Recently, investigations on the scope of CBD's medical benefits have encompassed its potential to treat substance use disorders. Both clinical and preclinical data are overall promising in this regard. However, presently available data are scattered across several classes of abused drugs (i.e., tetrahydrocannabinol, nicotine, opiates, alcohol) and several stages of the addiction cycle (i.e., selfadministration, withdrawal, drug seeking, abstinence). Moreover, both positive and negative findings have been obtained such that scope and nature of CBD's effects on addictive behavior remained to be more clearly established [2].

Among the studies of CBD's "anti-addiction" potential, a major positive finding reported by Hurd and colleagues [3] was that CBD attenuates cue-induced heroin reinstatement with effects that outlasted treatment by two weeks. Intrigued by these findings, we focused our efforts specifically on the anti-relapse potential of CBD and identified distinct leads in the literature suggestive of potential for several indications critical for relapse prevention. Many general behavioral effects of CBD, including anxiolytic, antistress, anti-depressant, and anti-compulsive actions (e.g., ref. [1]), are predictive of therapeutic benefit also for drug seeking and relapse. Moreover, CBD interacts with signaling mechanisms within the brain circuitry that regulates anxiety, drug desire associated with drug-related cues/contexts, and effects of stress on drug seeking. Lastly, CBD has both neuroprotective and proneurogenic actions [4]. The former includes attenuation of ethanol-induced neurodegeneration, an intoxication-induced deficit contributing to impaired impulse control in alcoholics. The proneurogenic effects of CBD have been implicated in the drug's anti-anxiety actions, and are predictive of anti-craving effects given emerging evidence implicating neurogenesis as an important factor in inhibiting drug seeking.

We therefore predicted that CBD may be suitable for targeting several relapse-promoting factors: craving associated with drug cue exposure, susceptibility to stress, heightened anxiety, and impaired impulse control. Testing this prediction in animal models of drug seeking (reinstatement), anxiety (elevated plus maze), and impulsivity (delay discounting), using rats with alcohol or cocaine self-administration histories, we found that CBD attenuated context-induced and stress-induced reinstatement of drug seeking without producing tolerance, sedative effects, or interfering with normal motivation. Following treatment termination, the attenuation of both context and stress-induced reinstatement remained unabated for the duration of the experiments (up to $\approx 5$ months). CBD also reduced experimental anxiety in rats with alcohol and cocaine histories, and prevented the development of high impulsivity in rats with a dependence-inducing alcohol intoxication history [5].

These findings reveal a profile of potential benefits of $C B D$ in relapse prevention that is unique in several respects: (1) Effects relevant for multiple vulnerability states that are often experienced concurrently by drug addicts and likely interact to exacerbate relapse risk. Therefore, the concurrent amelioration of these states by CBD is likely to be more effective in preventing relapse than treatment drug effects that target only a single state. (2) Long-lasting protective effects that far outlast treatment. Identification of mechanisms underlying these effects may lead improved understanding of neuroplasticity responsible for chronic susceptibility to relapse, as well as the development of more effective "anti-relapse" medications. (3) Efficacy across multiple drugs of abuse, that include not only cocaine and alcohol [5] but also opiates [3], including tentative anti-craving effects in early phase clinical trials [6]. Since co-abuse of opiates and cocaine with alcohol is common, the reported anti-reinstatement actions of CBD across three major classes of abused drugs further add to the putative treatment drug promise of this phytocannabinoid.

\section{ACKNOWLEDGEMENTS}

This is publication number 29746 from the Scripps Research Institute. This research was supported by NIH Grants AA022082 (FW), AA021549 (FW), and DA039821 (FW). Competing interests: The authors declare no competing interests.

Publisher's note: Springer Nature remains neutral with regard to jurisdictional claims in published maps and institutional affiliations.

\section{REFERENCES}

1. Campos AC, Moreira FA, Gomes FV, Del Bel EA, Guimaraes FS. Multiple mechanisms involved in the large-spectrum therapeutic potential of cannabidiol in psychiatric disorders. Philos Trans R Soc Lond B Biol Sci. 2012;367:3364-78.

2. Prud'homme M, Cata R, Jutras-Aswad D. Cannabidiol as an intervention for addictive behaviors: a systematic review of the evidence. Subst Abus. 2015;9:33-8.

3. Ren YH, Whittard J, Higuera-Matas A, Morris CV, Hurd YL. Cannabidiol, a nonpsychotropic component of cannabis, inhibits cue-induced heroin seeking and normalizes discrete mesolimbic neuronal disturbances. J Neurosci. 2009;29:14764-9.

4. Campos AC, Fogaca MV, Sonego AB, Guimaraes FS. Cannabidiol, neuroprotection and neuropsychiatric disorders. Pharmacol Res. 2016;112:119-27.

5. Gonzalez-Cuevas G, Martin-Fardon R, Kerr TM, Stouffer DG, Parsons LH, Hammell $\mathrm{DC}$, et al. Unique treatment potential of cannabidiol for the prevention of relapse to drug use: preclinical proof of principle. Neuropsychopharmacology. 2018;43:2036-45.

6. Hurd YL, Yoon M, Manini AF, Hernandez S, Olmedo R, Ostman M, et al. Early phase in the development of cannabidiol as a treatment for addiction: opioid relapse takes initial center stage. Neurotherapeutics. 2015;12:807-15.

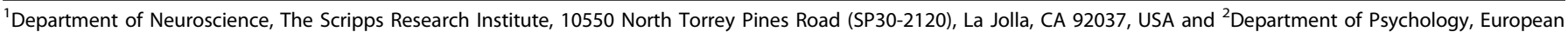
University of Madrid School of Biomedical Sciences, 28670 Madrid, Spain

Correspondence: Friedbert Weiss (bweiss@scripps.edu)
}

Published online: 25 September 2018 